\title{
Nuclear magnetic resonance study of alkane conformational statistics
}

\author{
E. Elliott Burnell, ${ }^{1, \text { a) }}$ Adrian C. J. Weber, ${ }^{1, b)}$ Cornelis A. de Lange, ${ }^{2, c)}$ \\ W. Leo Meerts, ${ }^{3, d)}$ and Ronald Y. Dong ${ }^{4, e)}$ \\ ${ }^{1}$ Chemistry Department, University of British Columbia, 2036 Main Mall, Vancouver, \\ British Columbia V6T 1Z1, Canada \\ ${ }^{2}$ Atomic, Molecular and Laser Physics, Vrije Universiteit, De Boelelaan 1081, 1081 HV Amsterdam, \\ The Netherlands \\ ${ }^{3}$ Institute for Molecules and Materials, Radboud University Nijmegen, Heyendaalseweg 135, \\ NL-6525 AJ Nijmegen, The Netherlands and Department of Physical Chemistry, Vrije Universiteit, \\ De Boelelaan 1083, 1081 HV Amsterdam, The Netherlands \\ ${ }^{4}$ Department of Physics and Astronomy, University of British Columbia, 6224 Agricultural Rd, Vancouver, \\ British Columbia V6T 1Z1, Canada
}

(Received 14 July 2011; accepted 10 November 2011; published online 19 December 2011)

\begin{abstract}
NMR spectra of ethane, propane, and $n$-butane as solutes in the nematic liquid crystals 4- $n$-pentyl4'-cyanobiphenyl (5CB) and Merck ZLI 1132 (1132) are investigated over a wide temperature range. The ratios of dipolar couplings of ethane to propane are constant over the entire temperature range. Assuming that this constancy applies to the butane conformers facilitates the separation of probability from order parameter. This separation allows the investigation of conformational distribution without the need of invoking any model for the anisotropic intermolecular potential. The results give an order matrix that is consistent with that predicted from model potentials that describe the orientational potential in terms of short-range size and shape effects. The isotropic intermolecular potential contribution to the trans-gauche energy difference $E_{t g}$ is found to be temperature dependent with the values and variation in agreement with that found when the same results are analyzed using the chord model for anisotropic interactions [A. C. J. Weber and E. E. Burnell, Chem. Phys. Lett. 506, 196 (2011)]. The fit obtained for 9 spectra in 5CB (63 dipolar couplings) has an RMS difference between experimental and calculated dipolar couplings of $2.7 \mathrm{~Hz}$, while that for the 16 spectra in 1132 (112 couplings) is $6.2 \mathrm{~Hz}$; this excellent fit with nine adjustable parameters suggests that the assumption of equal temperature dependencies of the order parameters for ethane, propane, and each conformer of butane is correct. Also the fit parameters $\left(E_{t g}\right.$ and the methyl angle increase) obtained for 1132 and $5 \mathrm{CB}$ agree. The results indicate that the chord model, which was designed to treat hydrocarbon chains, is indeed the model of choice for these chains. The temperature variation of $E_{t g}$ provides a challenge for theoreticians. Finally, even better fits to the experimental dipolar couplings are obtained when the energy in the Boltzmann factor is used for scaling ethane to butane results. However, in this case the values obtained for $E_{t g}$ differ between 1132 and 5CB. () 2011 American Institute of Physics. [doi:10.1063/1.3665139]
\end{abstract}

\section{INTRODUCTION}

The basic understanding of conformational statistics is crucial to a host of problems ranging from simple molecules to the macromolecules of biology. The study of solutes dissolved in nematic phases can provide information about solute geometries in the condensed phase through measurement and analysis of direct dipolar interactions. Initially there was, therefore, great hope that this method could lead to valuable information on conformational problems as well. However, so far these hopes have not been realized for a number of fundamental reasons.

\footnotetext{
a)Electronic mail: elliott.burnell@ubc.ca.

b) Electronic mail: adri@chem.ubc.ca. Present address: Chemistry Department, Brandon University, Brandon, Manitoba, Canada.

c) Electronic mail: c.a.de.lange@vu.nl.

d)Electronic mail: leo.meerts@ science.ru.nl. URL: http://www.leomeerts.nl.

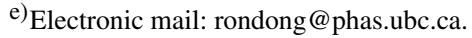

First, the interconversion between conformations is generally fast on the NMR time scale. Hence, averaging takes place on this time scale and separate NMR spectra of different conformers are usually not observed. Second, the description of the degree of orientational order of a solute in a nematic liquid crystal undergoing conformational change is far from trivial. A solute without symmetry dissolved in a nematic phase in a single conformation requires five independent orientational order parameters. Symmetry can reduce this number. For a solute undergoing conformational change it is important to realize that the time scale associated with the internal rearrangement of a significant part of the molecule cannot be very different from the time it takes for the solute as a whole to reorient in the anisotropic environment. Hence, the notion of an average solute is generally wrong and every conformation requires its own set of independent orientation parameters. ${ }^{1,2}$ This spells bad news because this increases the number of a priori unknown parameters in a very significant fashion. 
For rapid exchange among several rigid conformers $n$, the dipolar coupling between two nuclear spins $i$ and $j$ is

$$
D_{i j}=-\frac{h \gamma_{i} \gamma_{j}}{4 \pi^{2}} \sum_{n} p^{n} S^{i j, n} \frac{1}{r_{i j, n}^{3}}
$$

where we use the approximation (often utilized for a rigid solute) that assumes separation between internal vibrational motion and reorientation of the solute as a whole. Here $p^{n}$ represents the probability for the solute to find itself in conformation $n, S^{i j, n}$ reflects the degree of orientational order of conformer $n, r_{i j, n}$ is the internuclear distance between nuclei $i$ and $j$ in conformer $n, \gamma_{i}$ is the gyromagnetic ratio of nucleus $i$, and $h$ is Planck's constant.

The degree of orientational order of the vector connecting spins $i$ and $j$ for each separate conformer is given by

$$
S^{i j, n}=\left\langle\frac{3}{2} \cos ^{2} \theta_{i j, Z}^{n}-\frac{1}{2}\right\rangle .
$$

The angular brackets indicate averaging over the reorientational motion, with $\theta_{i j, Z}^{n}$ the angle between the $i j$ direction and the magnetic field. For the nematic liquid crystals used in the present study the director is coincident with the magnetic field direction $Z$.

Inspection of Eq. (1) shows that the conformer probability distribution $p^{n}$ in the condensed phase and the order tensor for each conformer $S^{i j, n}$ only occur as inseparable products. At best such products can be derived from experiment, but in order to make further progress additional information from an independent source, either about $p^{n}$ or about $S^{i j, n}$, is required. One is normally interested in the conformer probability distribution $p^{n}$ in the condensed phase. Although such a distribution can be estimated for an isolated molecule in the gas phase using sophisticated electronic structure calculations, the influence of its interactions in the condensed phase with the surrounding anisotropic environment cannot easily be calculated. Thus, the general approach has been to assume some model for the anisotropic interaction between solute and liquid-crystal environment, thus allowing one to relate the orientational order $S^{i j, n}$ among different conformers. Examples of models used for this purpose include those based on principal moments of inertia, ${ }^{3}$ an additive potential ${ }^{4}$ with extensions to account for overall solute shape in terms of a chord model, ${ }^{5-7}$ and interactions based on solute size and shape such as the circumference-integration (CI) model ${ }^{8,9}$ and the surface-potential model. ${ }^{10}$ The literature is replete with examples. Maximum entropy ideas ${ }^{11}$ have also been applied with some success to the conformational problem.

The problem with all these models is that they are phenomenological and that the quality of the predictions for $S^{i j, n}$ is hard to check and is different for each model. Moreover, with very few exceptions, it is difficult to assess reliably which model performs better than others. Hence, research groups often prefer their own models. This situation is an uneasy one, because the models used to predict $S^{i j, n}$ have an immediate impact on the values obtained for $p^{n}$. In a recent study of $n$-butane in nematic phases it was shown conclusively that the results obtained for $p^{n}$ were essentially modeldependent. ${ }^{12}$ It is clearly important to remedy this unfortunate situation.
In this paper, we present a different approach to this old problem by studying $n$-butane (butane hereafter) as a benchmark example. This molecule belongs to the alkanes and is the first member of the series that exhibits conformational change. In the simple rotameric state (RIS) model ${ }^{13}$ butane exists in either trans $(t)$, gauche $+\left(g^{+}\right)$or gauche- $\left(g^{-}\right)$ states. Butane has been studied before as a solute in nematic phases. ${ }^{14}$ With ten ${ }^{1} \mathrm{H}$ spins its spectrum is very complex and its analysis at the time proved to be an appreciable challenge that required appreciable NMR expertise. Since the introduction of covariance matrix adaptation evolutionary strategies (CMA-ES) to the problem of solutes with many spins in anisotropic environments, ${ }^{15-18}$ spectral analysis has become routine and is no longer the bottleneck in studies of this type. This allows for the first time an extensive temperature study of butane in nematic phases within reasonable time, with the aim of systematically changing the temperature-dependent Boltzmann factors that determine the conformer probability distribution.

Developing an understanding of the mechanisms that determine the degree of orientational order of a solute in an anisotropic environment has a long history. There is strong evidence that the orientational order of solutes in nematic phases can be described by two mechanisms, an electrostatic longrange one and a size-and-shape-dependent short-range one. ${ }^{19}$ The long-range mechanism is deemed to arise from the interaction between the solute molecular quadrupole moment and the average electric field gradient present in the anisotropic phase. ${ }^{20}$ This mechanism is considered relatively unimportant for zero electric-field gradient liquid-crystal mixtures, the socalled magic mixtures, or for solutes with small molecular quadrupole moments, the so-called magic solutes. Since alkanes have small electrostatic moments, butane as well as other alkanes can be considered magic solutes whose degree of orientational order is predominantly determined by the size-andshape mechanism. ${ }^{21}$

A temperature-dependent study of butane in itself does not lead to a separation of conformational probabilities and conformational order parameters. However, in the present study we combine temperature-dependent results for ethane and propane ${ }^{22}$ with those of butane. We will show how this approach leads to novel information about the butane conformational statistics without involving any model for either conformational statistics or conformational order parameters. This model-free approach can in principle be extended to higher alkanes and other solutes that undergo conformational change.

\section{EXPERIMENTAL}

The solutes $n$-butane and 1,3,5-trichlorobenzene (tcb) (added as an orientational reference to allow comparison of spectra taken with different samples) were co-dissolved to 5.0 , and $0.5 \mathrm{~mol} \%$ in the liquid-crystal solvent Merck ZLI1132 (1132) in one $5 \mathrm{~mm}$ o.d. standard NMR tube and in the liquid-crystal 4- $n$-pentyl-4'-cyanobiphenyl (5CB) in another. Since $n$-butane is a gas at room temperature and ambient pressure, it was allowed to flow into a vacuum and then condensed into an NMR tube which was pre-filled with liquid crystal 


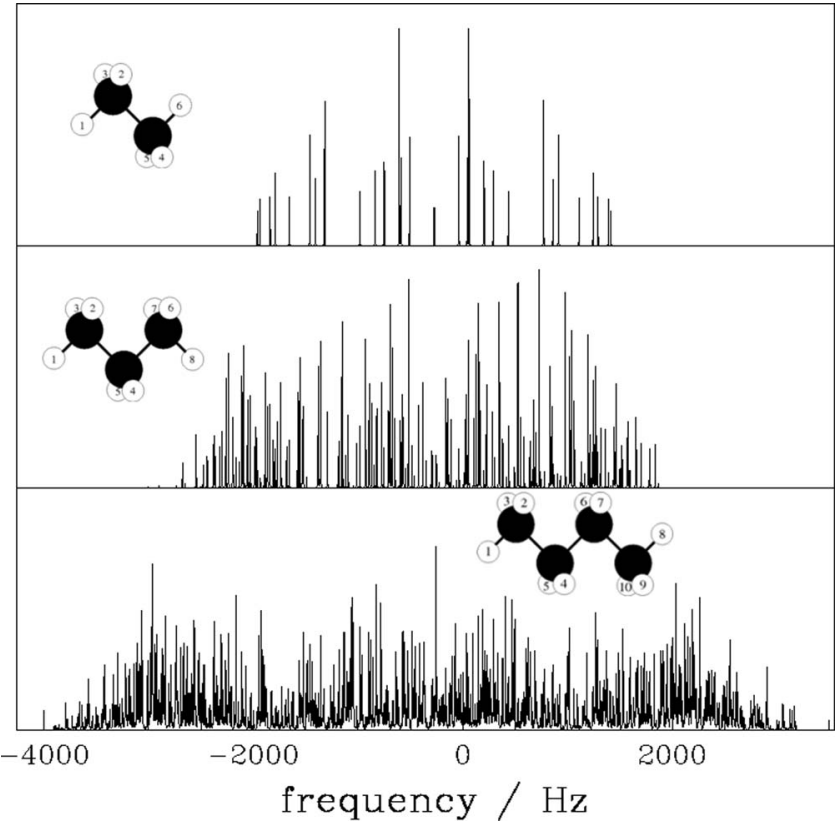

FIG. 1. Calculated NMR spectra of orientationally ordered ethane, propane and butane in $5 \mathrm{CB}$ at $298.5 \mathrm{~K}$.

and tcb and submerged into liquid nitrogen. After the sample was sealed and mixed thoroughly in the isotropic phase it was placed into a Bruker Avance $400 \mathrm{MHz}$ NMR spectrometer magnet. With the temperature controlled by the Bruker air-flow system, proton NMR spectra were acquired every $5^{\circ}$ from 253.5 to $333.5 \mathrm{~K}$ for the sample with butane in 1132 (Ref. 12) and from 263.5 to 298.5 for the sample with butane in 5CB. The spectral parameters defining the anisotropic spectra of all solutes are readily obtained with the use of a CMA-ES (Refs. 15-18) as can be seen in Figure 1 where it becomes obvious that the complexity of the spectra increases greatly with the number of nuclear spins.

GAUSSIAN03 (Ref. 23) was used to calculate the gasphase structure used for butane. Møller-Plesset second-order (MP2) perturbation theory ${ }^{24}$ was employed using Dunning's cc-pvdz basis set. ${ }^{25}$ The designated minima were confirmed to be minima by using analytical 1st and 2nd energy derivatives as is routine with MP2. For calculation of dipolar couplings, both methyl groups were rotated independently in $4^{\circ}$ steps in a threefold sinusoidal potential with $3006.12 \mathrm{cal} \mathrm{mol}^{-1}$ barrier.

The dipolar couplings for butane in 5CB are reported in Table I; the couplings for butane in 1132 are reported in
Ref. 12 and those for ethane and propane in both liquid-crystal solvents are reported in Ref. 22.

\section{RESULTS AND DISCUSSION}

We start with the RIS model for the conformers of butane where the molecule can exist in either a $t, g^{+}$or $g^{-}$state. The two gauche states are related by symmetry, and both the trans and gauche states have a $c_{2}$ symmetry axis (herein labelled y), meaning that their second-rank orientational order is described by the three independent order parameters $S_{x x}$, $S_{z z}$, and $S_{x z}$ for each conformer, giving six unknown order parameters in total. Note that the order tensor is traceless and thus $S_{y y}=-\left(S_{x x}+S_{z z}\right)$.

\section{A. Analysis of individual butane spectra, fit $p S$}

It is not possible for a given butane spectrum to determine separately the probabilities and order parameters in Eq. (1). However, it is possible to rewrite Eq. (1) in terms of order parameters $S_{\mu \nu}^{n}$ for molecular axes of conformer $n$ (the $\mathrm{z}$ axis is taken to be along the smallest principal moment of inertia (PMI) axis, $\mathrm{y}$ is the $\mathrm{c}_{2}$ axis and $x$ is perpendicular to $\mathrm{y}$ and $\mathrm{z}$ )

$$
S^{i j, n}=S_{\mu \nu}^{n} \cos \left(\theta_{i j, \mu}^{n}\right) \cos \left(\theta_{i j, \nu}^{n}\right),
$$

where $\theta_{i j, \mu}^{n}$ is the angle between the $i j$ and the molecule-fixed $\mu$ directions, and we use the Einstein convention that repeated Greek indices imply summation. We can then fit the six unknown products $p^{n} S_{\mu \nu}^{n}(n=t$ or $g)$ to the seven dipolar couplings obtained from a given butane spectrum.

However, a problem that frequently arises with methyl groups is the coupling between internal and reorientational motions which can lead to important nonrigid contributions to the dipolar couplings. ${ }^{26,27}$ These effects are notoriously difficult to predict, and are particularly severe for protons that are close together, as is the case of the methyl groups. For many cases in the past it has been found that this nonrigid effect leads to a seeming reduction in the methyl $\mathrm{CCH}$ angle. Hence, in order to account for these nonrigid effects, we also fit the decrease, $\triangle \mathrm{CCH}$, in the methyl $\mathrm{CCH}$ angle. Thus, because we fit seven unknowns to seven $D_{i j}$, we obtain an exact fit between experimental and calculated dipolar couplings for each butane spectrum recorded at all temperatures in both solvents 1132 and 5CB (fit $p S$ ). While we cannot separate $p^{n}$ from $S^{n}$, we can obtain two properties of the order

TABLE I. Experimental dipolar couplings in $\mathrm{Hz}$ of $n$-butane and tcb in $5 \mathrm{CB}$ as a function of temperature.

\begin{tabular}{|c|c|c|c|c|c|c|c|c|}
\hline$T(\mathrm{~K})$ & $D_{\mathrm{tcb}}$ & $D_{12}$ & $D_{14}$ & $D_{16}$ & $D_{18}$ & $D_{45}$ & $D_{46}$ & $D_{47}$ \\
\hline 263.5 & 229.66 & $1221.31 \pm 0.04$ & $-300.50 \pm 0.03$ & $-581.40 \pm 0.03$ & $-272.76 \pm 0.03$ & $2277.74 \pm 0.08$ & $121.02 \pm 0.12$ & $109.49 \pm 0.12$ \\
\hline 268.5 & 222.66 & $1158.61 \pm 0.04$ & $-283.53 \pm 0.03$ & $-554.06 \pm 0.03$ & $-262.50 \pm 0.03$ & $2178.59 \pm 0.08$ & $111.13 \pm 0.12$ & $95.59 \pm 0.12$ \\
\hline 273.5 & 213.81 & $1086.80 \pm 0.04$ & $-264.39 \pm 0.03$ & $-522.22 \pm 0.03$ & $-250.10 \pm 0.03$ & $2062.48 \pm 0.08$ & $100.47 \pm 0.12$ & $81.17 \pm 0.12$ \\
\hline 278.5 & 204.58 & $1014.05 \pm 0.04$ & $-245.34 \pm 0.03$ & $-489.36 \pm 0.03$ & $-236.82 \pm 0.03$ & $1941.41 \pm 0.08$ & $90.38 \pm 0.12$ & $67.81 \pm 0.12$ \\
\hline 283.5 & 194.04 & $939.99 \pm 0.04$ & $-226.33 \pm 0.03$ & $-455.47 \pm 0.03$ & $-222.53 \pm 0.03$ & $1815.10 \pm 0.07$ & $80.69 \pm 0.11$ & $55.90 \pm 0.11$ \\
\hline 288.5 & 181.46 & $860.01 \pm 0.04$ & $-206.13 \pm 0.03$ & $-418.25 \pm 0.03$ & $-206.29 \pm 0.03$ & $1674.94 \pm 0.07$ & $71.16 \pm 0.10$ & $44.65 \pm 0.10$ \\
\hline 293.5 & 166.75 & $770.85 \pm 0.04$ & $-183.94 \pm 0.03$ & $-376.20 \pm 0.03$ & $-187.33 \pm 0.03$ & $1514.61 \pm 0.07$ & $60.87 \pm 0.10$ & $34.76 \pm 0.10$ \\
\hline 298.5 & 147.08 & $662.84 \pm 0.04$ & $-157.53 \pm 0.03$ & $-324.42 \pm 0.03$ & $-162.92 \pm 0.03$ & $1313.23 \pm 0.07$ & $50.64 \pm 0.10$ & $24.17 \pm 0.10$ \\
\hline 303.5 & 104.78 & $454.22 \pm 0.02$ & $-107.52 \pm 0.02$ & $-223.08 \pm 0.02$ & $-113.08 \pm 0.02$ & $909.87 \pm 0.04$ & $32.95 \pm 0.06$ & $12.27 \pm 0.06$ \\
\hline
\end{tabular}


TABLE II. Parameters obtained from RIS fits to the temperature dependence of butane dipolar couplings

\begin{tabular}{|c|c|c|c|c|c|c|c|c|c|}
\hline Calculation and solvent ${ }^{\mathrm{a}}$ & RMS $(\mathrm{Hz})$ & $E_{t g}(300)\left(\mathrm{kcal} \mathrm{mol}^{-1}\right)$ & $\Delta \mathrm{CCH}(\mathrm{deg})$ & $\eta_{t}$ & $\theta_{t}(\operatorname{deg})^{\mathrm{b}}$ & $S_{z z}^{t}$ & $\eta_{g}$ & $\theta_{g}(\operatorname{deg})^{b}$ & $S_{z z}^{g}$ \\
\hline \multicolumn{10}{|l|}{ Model fits } \\
\hline Cd model ${ }^{\mathrm{c}}$ & 3.5 & 442 & 1.05 & 0.097 & -0.2 & 0.184 & -0.802 & 0.3 & 0.093 \\
\hline CI model ${ }^{c}$ & 7.8 & 676 & 1.43 & 0.144 & -1.4 & 0.164 & -0.428 & -0.2 & 0.108 \\
\hline \multicolumn{10}{|l|}{$p S$} \\
\hline $5 \mathrm{CB}$ & 0.0 & $\ldots$ & 1.313 & 0.334 & -5.017 & 0.218 & 5.176 & 2.692 & 0.040 \\
\hline 1132 & 0.0 & $\ldots$ & 1.226 & 0.305 & -5.038 & 0.370 & 4.927 & 3.601 & 0.069 \\
\hline \multicolumn{10}{|l|}{$S($ iso $)$} \\
\hline $5 \mathrm{CB}$ & 4.1 & $776(36)$ & $1.18(4)$ & 0.275 & -3.924 & 0.165 & 3.474 & 8.267 & 0.064 \\
\hline 1132 & 8.1 & $783(20)$ & $1.06(5)$ & 0.237 & -3.634 & 0.270 & 2.505 & -5.659 & 0.118 \\
\hline \multicolumn{10}{|l|}{$S$} \\
\hline $5 \mathrm{CB}$ & 3.9 & $560(71)$ & $1.02(5)$ & 0.207 & -1.920 & 0.167 & 1.068 & -2.124 & 0.067 \\
\hline 1132 & 8.0 & $552(26)$ & $0.94(4)$ & 0.179 & -2.003 & 0.275 & 0.841 & -1.423 & 0.119 \\
\hline \multicolumn{10}{|l|}{$S T^{\mathrm{d}}$} \\
\hline $5 \mathrm{CB}$ & 3.1 & $433(25)$ & $0.86(3)$ & 0.105 & 0.767 & 0.158 & -0.695 & -1.203 & 0.075 \\
\hline 1132 & 6.7 & $435(20)$ & $0.78(4)$ & 0.079 & 0.541 & 0.265 & -0.733 & -0.990 & 0.129 \\
\hline \multicolumn{10}{|l|}{$U$} \\
\hline $5 \mathrm{CB}$ & 3.4 & $517(20)$ & $0.98(3)$ & 0.215 & -1.241 & 0.166 & 0.621 & -2.394 & 0.068 \\
\hline 1132 & 7.7 & $419(15)$ & $1.01(4)$ & 0.214 & -2.376 & 0.308 & 1.388 & -3.849 & 0.101 \\
\hline \multicolumn{10}{|l|}{$U T^{\mathrm{e}}$} \\
\hline $5 \mathrm{CB}$ & 2.6 & $481(20)$ & $0.90(3)$ & 0.159 & 0.083 & 0.158 & -0.303 & -1.761 & 0.074 \\
\hline 1132 & 5.0 & $340(16)$ & $0.86(3)$ & 0.133 & -0.272 & 0.294 & -0.220 & -1.892 & 0.114 \\
\hline
\end{tabular}

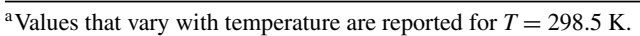

${ }^{\mathrm{b}} \theta$ is the angle between the PMI and POA axes, and is positive for clockwise rotation of the PMI axes about the $y \mathrm{c}_{2}$ symmetry axis for the structure displayed in Figure 1 . Note that the angle between the central CC bond and the PMI axis is $42.1^{\circ}$ for the trans and $23.2^{\circ}$ for the gauche conformer.

${ }^{\mathrm{c}}$ The RMS, $E_{t g}$ and $\Delta \mathrm{CCH}$ values are from fits to spectra taken at about $300 \mathrm{~K}$ of butane in four different liquid-crystal solvents using two-parameter chord or CI models for the orientational potential where the conformational problem included integration over the dihedral angle in $5^{\circ}$ steps. The order parameter information ( $\eta$, $\theta$, and $\left.S_{z z}\right)$ are for a fit to the spectrum in the magic mixture.

${ }^{\mathrm{d}} E_{1}\left(\mathrm{cal} \mathrm{mol}^{-1} \mathrm{~K}^{-1}\right)=-2.21 \pm 0.15$ for $5 \mathrm{CB}$ and $-2.00 \pm 0.11$ for 1132 .

${ }^{\mathrm{e}} E_{1}\left(\mathrm{cal} \mathrm{mol}^{-1} \mathrm{~K}^{-1}\right)=-1.38 \pm 0.10$ for $5 \mathrm{CB}$ and $-1.50 \pm 0.06$ for 1132

tensor $S^{n}$ : (i) the diagonalization angle $\theta_{n}$ (between the PMI and the diagonalized principal ordering axes, POA) which is not affected by multiplying all $S_{\mu \nu}^{n}$ by $p^{n}$ and (ii) the asymmetry $\left(\eta_{n}=\left(S_{x x}^{n}-S_{y y}^{n}\right) / S_{z z}^{n}\right)$ in the POA frame because it involves a ratio in which $p^{n}$ cancels. The results for spectra recorded at $298.5 \mathrm{~K}$ are reported in Table II.

At this stage we ask what would be reasonable values to expect for $\theta$ and $\eta$. Butane is considered to be a magic solute, and in a recent publication on butane in four different nematic solvents, two different models (both based on size and shape arguments) were used to rationalize the results. The parameter values obtained for one liquid-crystal solvent from these models are listed in Table II. Here $\theta$ values are small and hence the POA and PMI axes are virtually coincidental. Similar $\theta$ and $\eta$ values are anticipated from any correct analysis, and especially a negative value for $\eta_{g}$ is expected.

The $\theta$ values obtained for the individual fits, $p S$, are more or less reasonable, but the $\eta$ values disagree dramatically with those predicted by the size and shape model calculations. One result to note (not shown) is that the values obtained for $\eta$ and $\theta$ depend dramatically on the value chosen for the $\mathrm{CCH}$ angle decrease when it is fixed in the calculation. Potentially, these results do give us information on the location of the POA and the value of the order matrix asymmetry, although the results are problematic as they disagree with those obtained using models for the anisotropic interactions. More importantly, they do not allow separation of probability and orienta- tional order, and hence do not yield information on conformer statistics.

\section{B. Ethane and propane}

Next we turn to the dipolar coupling data ${ }^{22}$ obtained for ethane and propane as solutes. Can these be used to help sort out the butane problem? In order to investigate possibilities, we plot in Figure 2 (top panels) the ratios of the four propane dipolar couplings to the ethane intramethyl dipolar coupling $D_{i j}$ (propane) $/ D_{12}$ (ethane). We use the ethane intramethyl coupling because the inter-proton 1-2 direction (Figure 1) is perpendicular to the $c_{3}$ symmetry (z) axis and thus this coupling is directly proportional to $S_{z z}$. The other coupling, $D_{14}$, depends on the methyl rotation which is expected to have a slight temperature dependence. The result of the plot for both liquid crystals is very interesting: the ratio of propane to ethane dipolar couplings does not change significantly with temperature. The percent change for each coupling is given in the figure caption where it is seen that the maximum change is of order $3 \%$. The constancy of the propane to ethane ratio is striking.

\section{Details of the butane conformational problem}

The conformational distribution of butane in an anisotropic condensed phase is governed by three contributions to the Boltzmann factor. The first is the gas-phase 
intramolecular potential: we calculate this potential using GAUSSIAN03 (Ref. 23). The second is the isotropic interaction between solute and environment. We are not able to separate these first two terms and therefore must rely on the accuracy of the GAUSSIAN03 calculation in order to extract the intermolecular part. The third is the contribution from the anisotropic interaction, and when included, it is calculated as described below for each experiment from the order matrices which best reproduce the experimental dipolar couplings.

Thus the isotropic part of the potential, $U_{n}^{i s o}$, is composed of the intramolecular component, $U_{i n t, n}^{i s o}$ (which has been calculated with GAUSSIAN03), and the intermolecular part, $U_{\text {ext } n \text {. }}^{\text {iss }}$ Therefore, the trans-gauche energy difference, $E_{t g}$, has two contributions giving

$$
E_{t g}=U_{\text {gauche }}^{\text {iso }}-U_{\text {trans }}^{\text {iso }}=E_{\text {tg }}^{i n t}+E_{t g}^{\text {ext }},
$$

where $E_{t g}^{i n t} \equiv E_{t g}^{g a s}=643 \mathrm{cal} \mathrm{mol}^{-1}$ is taken as the energy difference between the minima of the potential wells of trans and gauche states as calculated by GAUSSIAN03. Values of $E_{t g}(T)$ are obtained for each experiment from fits to the experimental dipolar couplings (vide infra).

If one assumes a mean-field potential for orientational ordering of the solute, the order parameters of Eq. (3) can be written as

$$
S_{\mu \nu}^{n}=\frac{\int\left(\frac{3}{2} \cos \left(\theta_{\mu, Z}^{n}\right) \cos \left(\theta_{v, Z}^{n}\right)-\frac{1}{2} \delta_{\mu \nu}\right) \exp \left(-U_{n}^{\text {aniso }}(\Omega) / k T\right) d \Omega}{\int \exp \left(-U_{n}^{\text {aniso }}(\Omega) / k T\right) d \Omega}
$$

where $U_{n}^{\text {aniso }}$ is the anisotropic nematic ordering potential and $\theta_{\mu, Z}^{n}$ is the angle between the $\mu$-molecular axis and the nematic director which for experiments herein is aligned along the magnetic field $(Z)$ direction. Here $U_{n}^{\text {aniso }}$ is taken to be the classic Maier-Saupe potential ${ }^{28,29}$ which has been shown to be very successful for the description of the orientational order of solutes in nematic liquid crystals. ${ }^{30}$ For a solute of


FIG. 2. Ratio of propane dipolar couplings (top) and potential energy parameters (bottom) to the intramethyl dipolar couplings and the potential parameters of ethane in the liquid crystals $5 \mathrm{CB}$ (left) and 1132 (right) as a function of temperature. The maximum percent changes for $5 \mathrm{CB}$ are: $D_{12}$ of $0.4 \%, D_{14}$ of $2.0 \%, D_{16}$ of $0.3 \%, D_{45}$ of $1.2 \%, G \beta_{x x}$ of $0.8 \%$, and $G \beta_{y y}$ of $4.0 \%$, while those for 1132 are: $D_{12}$ of $0.9 \%, D_{14}$ of $2.9 \%, D_{16}$ of $0.6 \%, D_{45}$ of $1.9 \%$, $G \beta_{x x}$ of $0.7 \%$, and $G \beta_{y y}$ of $3.9 \%$. The x axis of propane is perpendicular to the plane of the three carbons and $y$ is the $c_{2}$ axis. general symmetry this potential can be written

$$
U_{n}^{\text {aniso }}=-\frac{3}{4} G_{Z Z}(\mathrm{LC}) \beta_{\mu \nu}(\text { solute }) \cos \left(\theta_{\mu, Z}^{n}\right) \cos \left(\theta_{\nu, Z}^{n}\right),
$$

where $G_{Z Z}(\mathrm{LC})$ is the $Z Z$ component of the anisotropic part of the liquid-crystal mean field and $\beta$ (solute) is the anisotropic part of some electronic molecular property.

Conversely, given values of $S_{\mu \nu}^{n}$ (e.g., from fitting the dipolar couplings) along with an assumed form of $U_{n}^{\text {aniso }}$, the parameters describing $U_{n}^{\text {aniso }}$ can be obtained from the fitted $S_{\mu \nu}^{n}$; then the desired probabilities $p^{n}$ can be calculated. Such a procedure requires iterations until a self-consistent solution of Eq. (5) is found.

The conformer probability is a function of both the isotropic $\left(U_{n}^{i s o}\right)$ and anisotropic $\left(U_{n}^{\text {aniso }}\right)$ parts of the potential and can be written

$$
p^{n}=\frac{G^{n} \exp \left(-U_{n}^{\text {iso }} / k T\right) \int \exp \left(-U_{n}^{\text {aniso }}(\Omega) / k T\right) d \Omega}{\sum_{n} G^{n} \exp \left(-U_{n}^{i s o} / k T\right) \int \exp \left(-U_{n}^{\text {aniso }}(\Omega) / k T\right) d \Omega},
$$

where $G^{n}=\sqrt{I_{x x}^{n} I_{y y}^{n} I_{z z}^{n}}$ is a rotational kinetic energy factor which is dependent on the principal values of the moment of inertia tensor for each conformer.

\section{Integration over dihedral angle}

The RIS approximation is obviously a crude approximation of the conformers available to butane. In order to increase the number of dihedral angles used in the calculation, it would appear necessary to include a new set of three adjustable $S$ parameters for each angle used in the integration. Since this cannot be done, an approximation is necessary. When dealing with small-amplitude vibrational internal motions, it is normal to separate the internal motion from the reorientation of the molecule as a whole. ${ }^{1,2,31}$ Thus we separate dihedral angles into two groups, one for conformers that fall in the trans and another for the gauche well. We assume that 
TABLE III. Parameters obtained from full potential fits (integration over all dihedral angles) to the temperature dependence of butane dipolar couplings

\begin{tabular}{|c|c|c|c|c|c|c|c|c|c|}
\hline Calculation and solvent ${ }^{\mathrm{a}}$ & RMS (Hz) & $E_{t g}(300)\left(\mathrm{kcal} \mathrm{mol}^{-1}\right)$ & $\triangle \mathrm{CCH}(\mathrm{deg})$ & $\eta_{t}$ & $\theta_{t}(\mathrm{deg})^{\mathrm{b}}$ & $S_{z z}^{t}$ & $\eta_{g}$ & $\theta_{g}(\mathrm{deg})^{\mathrm{b}}$ & $S_{z z}^{g}$ \\
\hline \multicolumn{10}{|l|}{$S($ iso $)$} \\
\hline $5 \mathrm{CB}$ & 4.0 & $747(34)$ & $1.23(4)$ & 0.271 & -4.578 & 0.170 & 4.292 & -1.972 & 0.059 \\
\hline 1132 & 7.7 & 751(20) & $1.11(4)$ & 0.237 & -4.302 & 0.279 & 3.181 & -13.874 & 0.109 \\
\hline \multicolumn{10}{|l|}{$S$} \\
\hline $5 \mathrm{CB}$ & 3.7 & $525(32)$ & $1.07(4)$ & 0.205 & -2.373 & 0.170 & 1.244 & -0.321 & 0.065 \\
\hline 1132 & 7.8 & $526(43)$ & $1.00(4)$ & 0.181 & -2.535 & 0.280 & 1.065 & 0.120 & 0.115 \\
\hline \multicolumn{10}{|l|}{$S T^{\mathrm{c}}$} \\
\hline $5 \mathrm{CB}$ & 2.7 & $387(21)$ & $0.90(3)$ & 0.098 & 0.751 & 0.159 & -0.840 & -0.714 & 0.076 \\
\hline 1132 & 6.2 & $405(18)$ & $0.84(3)$ & 0.076 & 0.403 & 0.265 & -0.824 & -0.501 & 0.130 \\
\hline \multicolumn{10}{|l|}{$U$} \\
\hline $5 \mathrm{CB}$ & 3.4 & 479(19) & $1.03(3)$ & 0.210 & -1.590 & 0.169 & 0.668 & -1.084 & 0.066 \\
\hline 1132 & 8.4 & $400(15)$ & $1.06(5)$ & 0.205 & -2.807 & 0.310 & 1.531 & -1.041 & 0.098 \\
\hline \multicolumn{10}{|l|}{$U T^{\mathrm{d}}$} \\
\hline $5 \mathrm{CB}$ & 2.2 & $424(13)$ & $0.94(2)$ & 0.148 & 0.061 & 0.160 & -0.474 & -1.069 & 0.073 \\
\hline 1132 & 4.5 & 299(14) & $0.90(3)$ & 0.123 & -0.347 & 0.295 & -0.362 & -1.147 & 0.114 \\
\hline
\end{tabular}

${ }^{\text {a }}$ Values that vary with temperature are reported for $T=298.5 \mathrm{~K}$

${ }^{\mathrm{b}} \theta$ is the angle between the PMI and POA axes, and is positive for clockwise rotation of the PMI axes about the y $\mathrm{c}_{2}$ symmetry axis for the structure displayed in Figure 1 . Note that the angle between the central CC bond and the PMI axis is $42.1^{\circ}$ for the trans and $23.2^{\circ}$ for the gauche conformer.

${ }^{\mathrm{c}} E_{1}\left(\mathrm{kcal} \mathrm{mol}^{-1} \mathrm{~K}^{-1}\right)=-2.59 \pm 0.14$ for $5 \mathrm{CB}$ and $-2.30 \pm 0.11$ for 1132 .

${ }^{\mathrm{d}} E_{1}\left(\mathrm{kcal} \mathrm{mol}^{-1} \mathrm{~K}^{-1}\right)=-1.73 \pm 0.06$ for $5 \mathrm{CB}$ and $-1.80 \pm 0.06$ for 1132 .

motions within the trans (or the gauche) well can be treated as vibrations and that such vibrations involve no conformer reorientation. In other words, we assume that within a particular potential energy well both the isotropic and anisotropic parts of the external potential do not change with internal vibrational motion. This is the first step toward separating internal motion from reorientation. In order to do this separation, we refer the order parameters to the PMI axes, and assume that the internal motion involves no reorientation of these axes. Thus, all conformers within a particular well have the same set of three order parameters. Of course the order matrix does change with potential-energy well. Thus, there will still be three adjustable trans and three adjustable gauche order parameters (or $G \beta$ energy parameters), as before. For each calculation of dipolar couplings we transform our original GAUSSIAN03 coordinates to these PMI axes. We next integrate (using Simpson's rule) over the dihedral angle in $5^{\circ}$ steps using gas-phase energies calculated from GAUSSIAN03 for $U_{i n t, n}^{i s o}(\phi)$. The dipolar coupling between spins $i$ and $j$ is then given by

$$
D_{i j}=-\frac{h \gamma_{i} \gamma_{j}}{4 \pi^{2}} \sum_{n} S_{\mu \nu}^{n} \sum_{\phi} p^{n, \phi}\left\langle\frac{1}{\left(r_{i j}^{n, \phi}\right)^{3}} \cos \left(\theta_{i j, \mu}^{n, \phi}\right) \cos \left(\theta_{i j, \nu}^{n, \phi}\right)\right\rangle,
$$

where the conformer probability $p^{n, \phi}$ now involves an additional contribution which depends on the dihedral angle $\phi$ in the Gaussian intramolecular potential $U_{i n t, n}^{i s o}(\phi)$ and $n=t$ or $g$,

$$
p^{n, \phi}=\frac{G^{n, \phi} \exp \left(-U_{\text {ext, }, \text { }}^{\text {iso }} / k T\right) \exp \left(-U_{\text {int }, n}^{\text {iso }}(\phi) / k T\right) \int \exp \left(-U_{n}^{\text {aniso }}(\Omega) / k T\right) d \Omega}{\sum_{n} \sum_{\phi} G^{n, \phi} \exp \left(-U_{\text {ext }, n}^{\text {iso }} / k T\right) \exp \left(-U_{\text {int }, n}^{\text {iso }}(\phi) / k T\right) \int \exp \left(-U_{n}^{\text {aniso }}(\Omega) / k T\right) d \Omega} .
$$

The sum over $\phi$ is taken in $5^{\circ}$ steps in the ranges where trans or gauche states are defined. The $\phi$ angles used are between $-60^{\circ}$ and $60^{\circ}$ for $t$ and between $65^{\circ}$ and $295^{\circ}$ for $g .{ }^{32}$ In Table II we report results of fits to the dipolar couplings for the RIS and in Table III for the full internal potential calculations. In Figure 3 we show the variation as a function of dihedral angle of the dipolar couplings for one of the calculations. Since vibrations, including the librational motion within a potential well about a $\mathrm{C}-\mathrm{C}$ bond, are treated as being separable from molecular rotations, the order parameters used for calculation of the dipolar couplings are kept constant for all dihedral angles within a given potential well. This leads to the discontinuity in dipolar couplings noted at the well maxima. We are treating butane as having two independent conformers, each with its own order matrix, with the order matrix being governed by interactions at or near the potential well minimum. The discontinuity is of no consequence because at the temperatures used for our experiments there is negligible population of dihedral angles near the well maxima. 


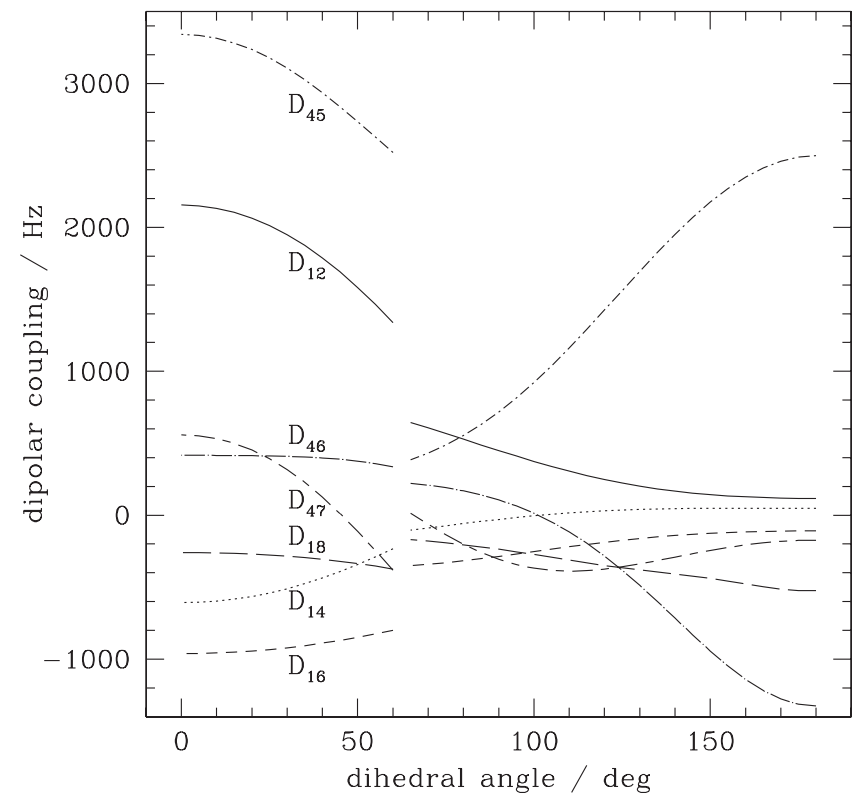

FIG. 3. Calculated $D_{i j}$ for butane in 1132 as a function of dihedral angle (for the full potential fit $S T$ at $T=293.5 \mathrm{~K}$ ). The order parameters from the fit (that are used to calculate these couplings) are: $S_{z z}^{t}=0.2797$, $S_{x x}^{t}=-0.1292, S_{x z}^{t}=-0.0029, S_{z z}^{g}=0.1367, S_{x x}^{g}=-0.1247$, and $S_{x z}^{g}$ $=0.0023$. There is a discontinuity in dipolar couplings at $60^{\circ}-65^{\circ}$ because these angles give a maximum in the potential energy and hence are used as the division between trans and gauche conformers which have quite different order parameters. The conformer population at these angles is very low because of the high potential energy.

\section{E. Butane dipolar couplings scaled to ethane intramethyl dipolar couplings, fit $\boldsymbol{S}$ (iso)}

The invariance of the propane/ethane dipolar coupling ratios suggests a very interesting possibility. Could it be that for hydrocarbons the dipolar couplings for any given conformer will be proportional to the ethane dipolar couplings in the same environment? We here make the assumption that this is indeed the case, and explore the consequences for the analysis of the butane results. This assumption is consistent with the idea that hydrocarbons are magic solutes and that their orientational ordering arises from size and shape effects alone. In other words, we assume that for these magic solutes, the ordering in the liquid-crystal environment depends on a single mechanism that only involves solute size and shape, and that for alkanes in general all elements of the order matrix (for example, the three independent parameters for each of the trans and gauche conformers of butane) should scale the same way to ethane as they do for propane (two independent order parameters) to ethane (one independent order parameter).

As the butane spectra were recorded using a different sample tube, we must find the $D_{12}$ (ethane) for the same conditions as apply to the butane spectra. For this purpose tcb was dissolved in all samples in order to provide an orientational reference. We use a spline fit of the tcb versus ethane couplings in order to use the tcb coupling in the butane sample to calculate the ethane coupling for the butane sample. Figure 4 gives the variation of $D_{i j}$ (butane)/ $/ \mathrm{D}_{12}$ (ethane) as a function of temperature for both liquid crystals. As can be seen, the ratios are not constant, which is expected as a direct

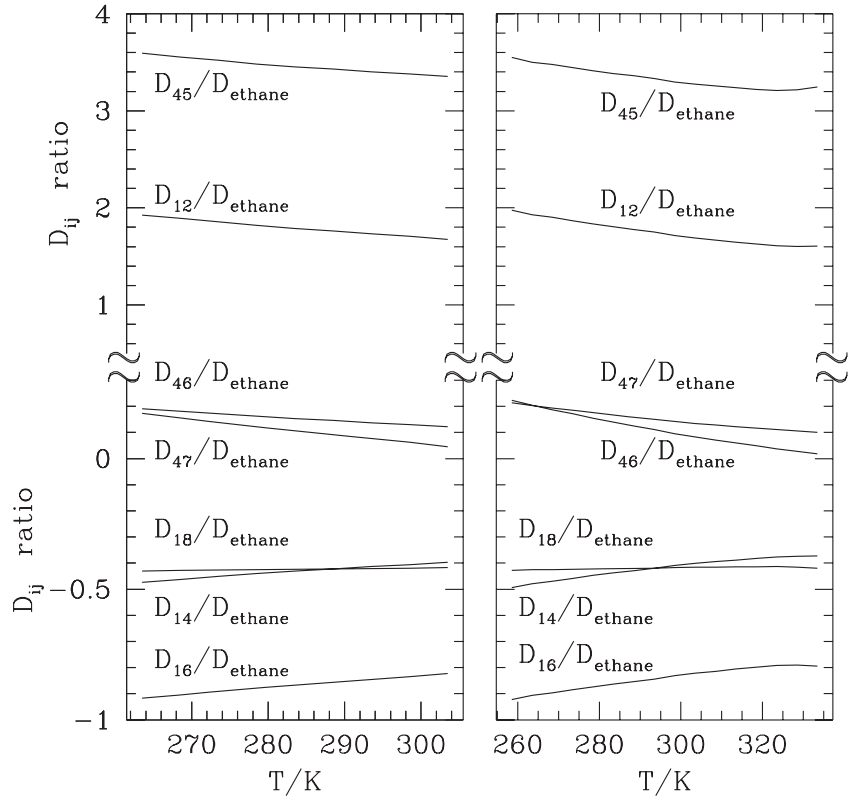

FIG. 4. Ratio of butane dipolar couplings to the intramethyl dipolar couplings of ethane in the liquid crystal 5CB (left) and 1132 (right) as a function of temperature. Note the change in scale of the $y$ axis.

result of changes in conformer populations as a function of temperature.

Hence, we now assume that each of the six unknown order parameters in butane (three for trans and three for gauche) for each temperature scale to the ethane dipolar coupling (i.e., order parameter) for that temperature. This means that we fit all spectra in a given liquid crystal to six $\Gamma$ constants, and the order parameters for butane are then $S_{\mu \nu}$ $=\Gamma_{\mu \nu} \times D_{12}$ (ethane) $/ 1000$ for that temperature. In these calculations, labelled S(iso) in Tables II and III, besides the six $\Gamma$ constants, the $\mathrm{CCH}$ angle decrease and $E_{t g}$ are also fitted. Note that in this case, $p^{n}$ in Eq. (7) and $p^{n, \phi}$ in Eq. (9) are used with $U_{n}^{\text {aniso }}=0$. Quite reasonable fits with eight unknowns are obtained from nine spectra of butane in $5 \mathrm{CB}$, and 16 spectra of butane in 1132, giving RMS differences between experimental and recalculated dipolar couplings of 4.0 and $7.7 \mathrm{~Hz}(4.1$ and $8.1 \mathrm{~Hz}$ when the RIS approximation is used). This is remarkable agreement - especially considering that the RMS is equal to or better than the best fits found in earlier studies where couplings from a single spectrum were fitted using some model for calculation of order parameters. ${ }^{12,14,32}$

The $\Gamma$ constants can be diagonalized, and the $\eta$ and $\theta$ values obtained pertain to the order matrix. As was the case for the order parameters in the fit $p S$ calculation above, the $\theta$ values indicate that PMI and POA axes do not differ greatly, but $\eta$ values for the gauche conformer are still inconsistent with intuition based on size and shape arguments (see Tables II and III).

\section{F. The anisotropic potential partition function, fit $S$}

While the $S$ (iso) calculation does account for the possibility that the condensed medium can give rise to an isotropic contribution to the energy difference between trans and 
gauche states, it does not take into account the change in probability caused by the anisotropic part of the intermolecular potential. In earlier calculations that used models for the anisotropic intermolecular potential, the anisotropic contribution to conformer probabilities is accounted for by the partition function for the anisotropic part of the potential,

$$
Z_{n}^{\text {aniso }}=\int \exp \left(-U_{n}^{\text {aniso }}(\Omega) / k T\right) d \Omega .
$$

The way we have managed to deal with this is as follows. In our fit to the $D_{i j}$, we need to use $\Gamma$ constants as adjustable least-squares parameters, and these multiplied by the ethane $D_{12}$ (ethane) give the order parameters. To calculate the $Z_{n}^{\text {aniso }}$ associated with these order parameters is not straightforward (the reverse would be). Thus, every time in the least squares when we calculate dipolar couplings from the variable $\Gamma$ components, we call a second routine to fit the conformer order parameters $S_{x x}, S_{z z}$, and $S_{x z}$ to Maier-Saupe energy parameters $G \beta_{x x}, G \beta_{z z}$, and $G \beta_{x z}$ (this gives an exact fit). This calculation yields the required $Z_{n}^{\text {aniso }}$, and this $Z_{n}^{\text {aniso }}$ is then used in the fit to the $D_{i j}$. We emphasize that the only thing we use from this second routine is the value of $Z_{n}^{\text {aniso }}$.

Using this approach, we see in Table III that the RMS errors are now 3.7 and $7.8 \mathrm{~Hz}(3.9$ and $8.0 \mathrm{~Hz}$ for the RIS calculation in Table II), essentially the same as before, but $E_{t g}^{\text {ext }}$ is about $200 \mathrm{cal} \mathrm{mol}^{-1}$ smaller and $\eta$ values are smaller, getting closer to values expected from size and shape arguments - but $\eta_{g}$ is still positive.

\section{G. Temperature variation of $E_{t g}^{e x t}$, fit $S T$}

The butane data for 1132 were reported previously ${ }^{12}$ where they were analyzed in terms of models for the anisotropic interactions. It was found that the results obtained depend on the model used. One of the models, the chord model, resulted in a temperature dependence of $E_{t g}$ while the other, the CI model, gave either a slight or no temperature variation of this parameter. In addition, the value of $E_{t g}$ obtained depended on model. Hence, we first add the possibility of a linear temperature variation of $E_{t g}$ and write

$$
E_{t g}^{e x t}(T)=E_{t g}^{e x t}(300)+E_{1}(T-300 \mathrm{~K}) .
$$

This has a dramatic effect. Not only is the RMS reduced to 2.7 and $6.2 \mathrm{~Hz}$, but $\eta_{g}$ is now -0.8 in agreement with model predictions. Also the principal ordering axes lie very close to the PMI axes.

It is interesting to compare our fits with the results of the earlier paper on 1132 in which models were used for the analysis of each individual temperature. Figure 5 compares the $E_{t g}$ values found here with those from the chord model in the previous work, and it is seen that the agreement is remarkable. Note that the present calculation fits to all dipolar couplings (63 for 5CB and 112 for 1132) with six adjustable $\Gamma$ constants, one $\mathrm{CCH}$ angle decrease and up to two $E_{t g}$ parameters $\left(E_{t g}(300)\right.$ and $\left.E_{1}\right)$. It is remarkable that the RMS obtained for the linear temperature fit is $2.7 \mathrm{~Hz}(5 \mathrm{CB})$ and $6.2 \mathrm{~Hz}(1132)-$ this result is strong indication of the validity of the assumption that order parameters for butane conformers scale to ethane

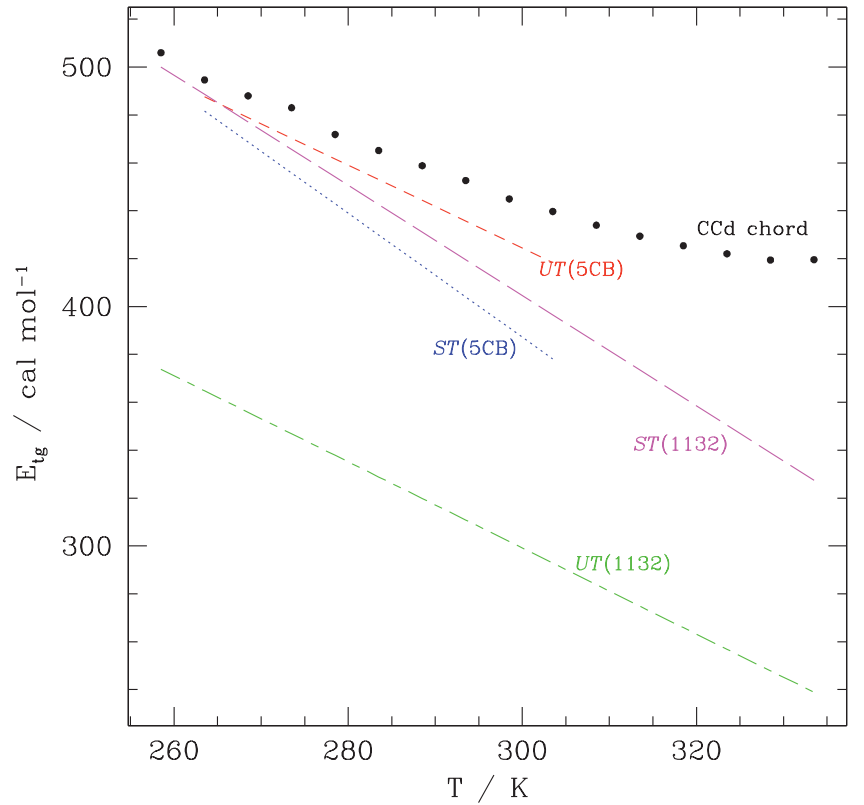

FIG. 5. $E_{t g}$ as a function of temperature where "CCd chord" is the modified chord model fit to $1132 .{ }^{12}$

dipolar couplings. Such a small RMS difference is unprecedented in earlier studies of orientationally ordered hydrocarbons. In addition, the present analysis does not rely on any model for the anisotropic intermolecular potential, but only on the scaling of order parameters. It is also encouraging that as we add physical intuition to the calculation, the fit gets better, and the parameters obtained (especially $\eta_{g}$ which for the linear-temperature fit to 1132 is -0.840 compared to -0.802 for the $\mathrm{Cd}$ model) become more in line with that expected from size and shape arguments which are expected to dominate the orientational order of hydrocarbons. Our results are clearly consistent with a temperature variation in $E_{t g}^{e x t}$, and this result provides a challenge for theoreticians.

The values obtained for $E_{t g}$ for all calculations that include the contribution of the anisotropic potential are all considerably lower than the GAUSSIAN03 gas-phase value of $643 \mathrm{kcal} \mathrm{mol}^{-1}$. This agrees with earlier studies where the effect of the condensed phase is found both theoretically ${ }^{33,34}$ and experimentally (see Refs. 14,32, and references therein) to lower the value of $E_{t g}$. Hence, the condensed phase favours the gauche conformer, which can be interpreted in terms of packing effects on the entropy of the gauche being greater than that for the trans.

The effect of the anisotropic intermolecular potential $U_{n}^{\text {aniso }}$ on conformer populations is displayed in Figure 6 for the full intramolecular fit $S T$ where the populations calculated for the isotropic potential $U_{n}^{i s o}$ and the total potential $U_{n}^{i s o}+U_{n}^{\text {aniso }}$ are shown. It is clear that the anisotropic contribution leads to an increase in the trans population, and that the effect is larger at lower temperature where the conformer orientational order is larger.

The $G \beta_{\mu \nu}$ (butane) potential parameter values calculated from the order parameters give the ratios of $G \beta_{\mu \nu}$ (butane) $/ G \beta$ (ethane) as displayed by the points in Figure 7 , left for 5CB and right for 1132 . While a slight variation 


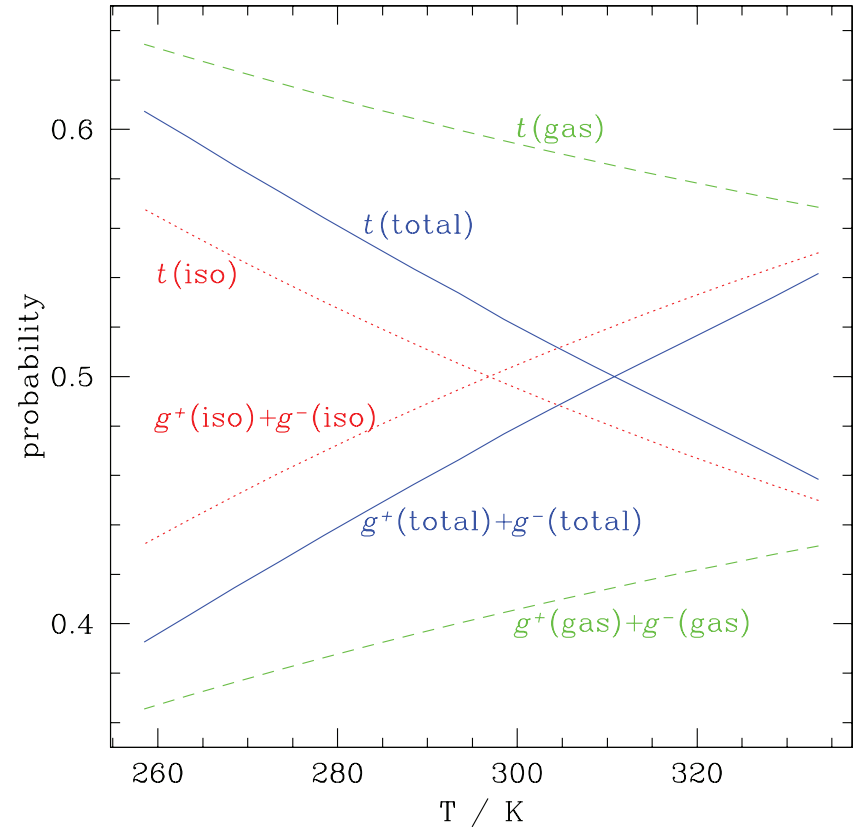

FIG. 6. Temperature dependence of butane trans and gauche conformer probabilities from Table III fit $S T$ where $n$ (total) is the probability of conformer $n$ (which includes $U_{n}^{\text {iso }}$ and $U_{n}^{\text {aniso }}$ contributions to the Boltzmann factor) and where $n($ iso $)$ is the probability calculated if we ignore the anisotropic contribution. The gas-phase probabilities calculated from Eq. (9) (setting $U_{\text {ext }, n}^{i s o}=0$ and $U_{n}^{\text {aniso }}(\Omega)=0$ ) are also shown. The hightemperature limit is $p_{t}=p_{g^{+}}=p_{g^{-}}=\frac{1}{3}$.

with temperature is noted, the values are essentially constant, indicating that scaling with either order parameter or with the anisotropic potential parameter should give essentially equivalent results.

\section{H. Scaling to anisotropic potential parameters, fits $U$ and $U T$}

Propane and ethane have quite different orientational order, and one would expect that it would be the ratio of the anisotropic intermolecular potentials that would be constant. We note that in an earlier study on scaling among samples of differing concentrations it was found best to scale tcb order parameters and not potential parameters. ${ }^{35}$ We also note in Figure 2 (bottom panels) that the $G \beta$ ratios are also independent of temperature.

The orientational order involves $G(\mathrm{LC}) \beta$ (solute), and logic (mean-field) would seem to suggest that changing liquid-crystal conditions (via temperature or composition) should change the $G(\mathrm{LC})$ part only. Hence scaling using $G \beta$ instead of the measured $S$ (that can be used to calculate a Maier-Saupe $G \beta$ ) should work. To be complete, here we investigate ethane potential scaling for the butane problem.

Thus, we repeat $S$ and $S T$ calculations by scaling the butane $G \beta_{\mu \nu}$ parameters to $G \beta$ (ethane) values (fits $U$ and $U T$ ). Now the calculation of $Z_{n}^{\text {aniso }}$ is done automatically in the fit. The results are in Tables II and III. In general, the RMS is improved, and the results make as much sense as for order parameter scaling. The only problem is that $E_{t g}$ obtained for 1132 when a linear temperature dependence (fit UT) is imposed is rather lower than values obtained in the other calcula-
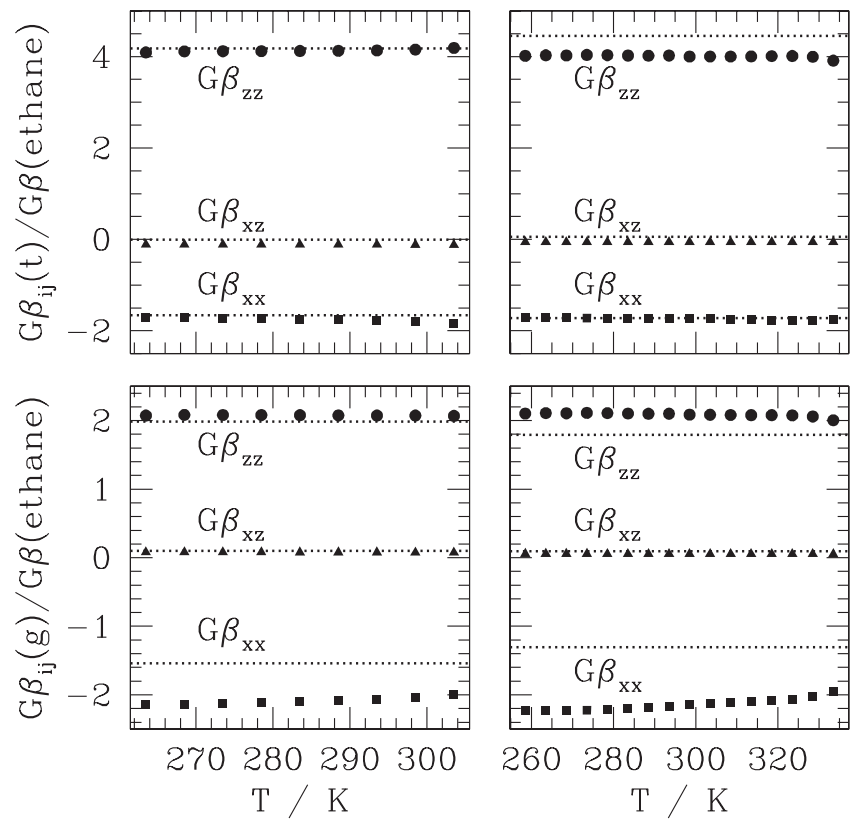

FIG. 7. Ratio of butane potential parameters to those of ethane as a function of temperature in 5CB (left) and in 1132 (right). The points are values from fits $S T$ which include a linear temperature dependence of $E_{t g}$. The lines are the constant values obtained from the fits $U T$ (scaling to anisotropic potential parameters with a linear temperature dependence of $E_{t g}$ ). The $\mathrm{z}$ axis is the PMI axis with lowest moment of inertia, $y$ is the $c_{2}$ axis and $x$ is perpendicular to $\mathrm{y}$ and $\mathrm{z}$.

tions, and when compared to values reported in the literature. The $1132 \eta_{g}$ value for linear temperature dependence $(-0.36)$ agrees with the CI model value.

The $G \beta_{\mu \nu}$ (butane) $/ G \beta$ (ethane) values obtained when assuming a linear temperature dependence of $E_{t g}(U T)$ are given as dotted lines in Figure 7 for direct comparison with those found for $S T$. In all cases, $G \beta_{x z}$ (butane) $/ G \beta$ (ethane) is small indicating that the principal axes of the $\beta$ tensor lie close to the PMI axes.

The values of all ratios in Figure 7 from scaling to the ethane potential are in quite reasonable agreement with those calculated from the order-parameter scaling. As both orderparameter and potential scalings apply to propane/ethane spectra, it is pleasing to see that both give almost equivalent results for the butane spectra, with the exception that order parameter scaling does give the same results for 1132 and 5CB, whereas potential scaling has problems with 1132 (quite low value of $E_{t g}$ ) while for 5CB it gives essentially the same result as for order parameter scaling (see Figure 7).

\section{CONCLUSION}

In this paper we obtain information about the conformational averaging of butane in an orientationally ordered condensed phase. We note that as we improve the details of the calculation, we get not only lower RMS errors, but also parameters that make more physical sense. The RMS for the calculations involving integrating over all dihedral angles using molecular energies calculated with GAUSSIAN03 is lower than for the same calculations using the three-state RIS approximation. This RMS decreases when we include 
the anisotropic energy contribution to the conformer probabilities, and further decreases when we include a linear temperature dependence of $E_{t g}$. Indeed an RMS of $6.2 \mathrm{~Hz}$ for fitting sixteen 1132 spectra, and $2.7 \mathrm{~Hz}$ for fitting nine 5CB spectra is "spectacular"! Consider what we do - we assume that each of the three trans and the three gauche order parameters are equal to a constant times the $D_{12}$ (ethane), and thus we vary only eight or nine parameters being: the $\Gamma_{x x}, \Gamma_{z z}$, and $\Gamma_{x z}$ parameters for each of trans and gauche conformers, $E_{t g}(300)$, $E_{1}$ and the $\mathrm{CCH}$ angle decrease. Plots of $E_{t g}$ versus $T$ (Figure 5) look remarkably similar to the plot using the chord model in Refs. 12 and 36.

The parameters obtained from this linear temperature fit make excellent physical sense. The diagonalization angles $\theta_{t}$ and $\theta_{g}$ are both quite small, indicating that the principal ordering axes for both conformers lie close to the principal inertial axes. Of course there is no reason why the principal ordering and principal inertial axes should correspond. However, in the sense that the PMI can be taken as some sort of crude approximation of molecular shape, their proximity is a pleasing result.

The asymmetry $\eta$ of the diagonalized order matrix is quite an interesting number. For calculations where we do not include a temperature dependence of $E_{t g}$ the typical values we obtain are $\eta_{t} \approx 0.2$ and $\eta_{g}>0$. The values from models for short-range size and shape interactions are $\eta_{t} \approx 0.1$ and $\eta_{g} \approx-0.6$. Of particular note here is that $\eta_{g}$ is negative for calculations involving models. In our present fits, we only get this negative result when we include a temperature dependence of $E_{t g}$. If orientational order is governed by size and shape (which we strongly expect to be the case, especially for hydrocarbons ${ }^{19,21}$ ), then $\eta$ should be consistent with size and shape arguments which are the bases of the chord and CI model calculations in Ref. 32.

The physics in the fits includes: $4^{\circ}$ steps for methyl rotation; the full potential for the dihedral angle; a possible temperature dependence of $E_{t g}=E_{t g}^{i n t}+E_{t g}^{\text {ext }} ; S_{\mu \nu}$ (butane, $T$ ) $=D_{12}($ ethane, $T) \times \Gamma_{\mu \nu} / 1000$ where six adjustable $\Gamma_{\mu \nu}$ orientational parameter factors are used to fit all 9 or 16 spectra; tcb splittings used to interpolate $D_{12}$ (ethane, $T$ ) values; the contribution to the Boltzmann factor from the anisotropic potential (by calculating the partition function when we fit the $S_{\mu \nu}$ (butane) to Maier-Saupe $G \beta_{\mu \nu}$ parameters) which leads to an increase in the trans population that is larger with higher orientational order (Fig. 6); and a decrease in the $\mathrm{CCH}$ angle to account for the nonrigid effect that is often found for intramethyl dipolar couplings. Thus, we stress, when we include what we feel is all the physics in the best possible manner we obtain: the reasonable value of $0.9^{\circ}$ for the $\mathrm{CCH}$ angle decrease; the principal ordering and inertial axes lie very close to each other; the asymmetry in the order matrices are in excellent agreement with size and shape model calculations; and an RMS of 6.2 (1132) and $2.7(5 \mathrm{CB}) \mathrm{Hz}$ is obtained in the $S T$ calculations, these numbers being phenomenal considering that we are fitting 9 or 16 spectra over a $40^{\circ}$ or $75^{\circ}$ temperature range.

\section{ACKNOWLEDGMENTS}

We acknowledge financial support from the Natural Sciences and Engineering Research Council of Canada.

${ }^{1}$ E. E. Burnell and C. A. de Lange, Chem. Phys. Lett. 76, 268 (1980).

${ }^{2}$ E. E. Burnell and C. A. de Lange, J. Magn. Reson. 39, 461 (1980).

${ }^{3}$ E. T. Samulski and R. Y. Dong, J. Chem. Phys. 77, 5090 (1982).

${ }^{4}$ R. Y. Dong, Nuclear Magnetic Resonance of Liquid Crystals, 1st ed. (Springer-Verlag, New York, 1994).

${ }^{5}$ D. J. Photinos, E. T. Samulski, and H. Toriumi, J. Phys. Chem. 94, 4688 (1990).

${ }^{6}$ D. J. Photinos, E. T. Samulski, and H. Toriumi, J. Phys. Chem. 94, 4694 (1990)

${ }^{7}$ A. G. Vanakaras and D. J. Photinos, Mol. Cryst. Liq. Cryst. 262, 463 (1995).

${ }^{8}$ A. J. van der Est, M. Y. Kok, and E. E. Burnell, Mol. Phys. 60, 397 (1987).

${ }^{9}$ D. S. Zimmerman and E. E. Burnell, Mol. Phys. 78, 687 (1993).

${ }^{10}$ A. Ferrarini, G. J. Moro, P. L. Nordio, and G. R. Luckhurst, Mol. Phys. 77, 1 (1992).

${ }^{11}$ D. Catalano, L. Di Bari, C. A. Veracini, G. N. Shilstone, and C. Zannoni, J. Chem. Phys. 94, 3928 (1991).

${ }^{12}$ A. C. J. Weber and E. E. Burnell, Chem. Phys. Lett., 506, 196 (2011). (Note that the $\mathrm{CCH}$ angle increase reported in this paper is actually the $\mathrm{CCH}$ angle decrease).

${ }^{13}$ P. J. Flory, Statistical Mechanics of Chain Molecules (Wiley-Interscience, New York, 1969).

${ }^{14}$ J. M. Polson and E. E. Burnell, J. Chem. Phys., 103, 6891 (1995).

${ }^{15}$ W. L. Meerts and M. Schmitt, Int. Rev. Phys. Chem., 25, 353 (2006).

${ }^{16}$ W. L. Meerts, C. A. de Lange, A. C. J. Weber, and E. E. Burnell, J. Chem. Phys., 130, 044504 (2009), and references therein.

${ }^{17}$ A. C. J. Weber, X. Yang, R. Y. Dong, W. L. Meerts, and E. E. Burnell, Chem. Phys. Lett., 476, 116 (2009).

${ }^{18}$ E. E. Burnell, C. A. de Lange, and W. L. Meerts, Nuclear Magnetic Resonance Spectroscopy of Liquid Crystals, edited by R. Y. Dong (World Scientific, Singapore, 2010), p. 1.

${ }^{19}$ NMR of Ordered Liquids, edited by E. E. Burnell and C. A. de Lange (Kluwer Academic, Dordrecht, 2003).

${ }^{20}$ E. E. Burnell and C. A. de Lange, Chem. Rev. 98, 2359 (1998), and references therein.

${ }^{21}$ A. F. Terzis, C.-D. Poon, E. T. Samulski, Z. Luz, R. Poupko, H. Zimmermann, K. Müller, H. Toriumi, and D. J. Photinos, J. Am. Chem. Soc. 118, 2226 (1996).

${ }^{22}$ A. C. J. Weber, D. H-J Chen, and E. E. Burnell, "NMR of ethane and propane in nematic liquid crystals" (unpublished).

${ }^{23}$ H. Nakatsuji, M. Hada, M. Ehara et al., GAUSSIAN 03, Revision E.01, Gaussian, Inc., Wallingford, CT, 2004.

${ }^{24}$ C. Møller and M. S. Plesset, Phys. Rev. 46, 618 (1934).

${ }^{25}$ T. H. Dunning, J. Chem. Phys. 90, 1007 (1989).

${ }^{26}$ E. E. Burnell, C. A. de Lange, J. B. S. Barnhoorn, I. Aben, and P. F. Levelt, J. Phys. Chem. A. 109, 11027 (2005).

${ }^{27}$ C. A. de Lange, W. L. Meerts, A. C. J. Weber, and E. E. Burnell, J. Phys. Chem. A. 114, 5878 (2010).

${ }^{28}$ W. Maier and A. Saupe, Z. Naturforsch. A. 14, 882 (1959).

${ }^{29}$ W. Maier and A. Saupe, Z. Naturforsch. A. 15, 287 (1960).

${ }^{30}$ E. E. Burnell, L. C. ter Beek, and Z. Sun, J. Chem. Phys., 128, 164901 (2008). Note that the $S_{x x}$ values for fluorobenzene in 1132 and MM reported in Table I should both be positive.

${ }^{31}$ E. E. Burnell, C. A. de Lange, and O. G. Mouritsen, J. Magn. Reson., 50, 188 (1982)

${ }^{32}$ A. C. J. Weber, C. A. de Lange, W. L. Meerts, and E. E. Burnell, Chem. Phys. Lett. 496, 257 (2010).

${ }^{33}$ L. R. Pratt, C. S. Hsu, and D. Chandler, J. Chem. Phys. 68, 4202 (1978).

${ }^{34}$ C. S. Hsu, L. R. Pratt, and D. Chandler, J. Chem. Phys. 68, 4213 (1978).

${ }^{35}$ R. T. Syvitski, M. Y.-M. Pau, and E. E. Burnell, J. Chem. Phys. 117, 376 (2002)

${ }^{36}$ A. C. J. Weber, Ph.D. dissertation, University of British Columbia 2010. 